\title{
LETTRES D'HENRI POINCARÉ À L. FUCHS.'
}

I.

Caen, le 29 Mai r 880.

Monsieur le Professeur,

J'aj lu avec le plus grand intérêt le remarquable mémoire que vous avez fait insérer dans la dernière livraison du Journal de Crelle ${ }^{2}$ et qui a pour titre: Ueber die Verallgemeinerung des Umkehrproblems. Veuillez me permettre, Monsieur, de vous demander au sujet de ce travail, quelques éclaircissements.

Vous démontrez, page I59 que la fonction $z$ est fonction méromorphe de $\zeta$, toutes les fois que $\zeta$ prend une valeur correspondant à une valeur donnée de $z$; que cette valeur de $z$ soit un point ordinaire ou un point singulier, qu'elle soit finie ou infinie. Vous démontrez ensuite, page $I 60$ que cela est encore vrai pour $\zeta=\infty$ et comme conclusion vous dites:

.... so ist die durch die Gleichung (H.) definirte Function $z$ von $\zeta$ für alle Werthe von $\zeta$ meromorph.

Il s'agit ici de toutes les valeurs de $\zeta$ finies et infinies; cet énoncé ferait donc entendre que $z$ est fonction méromorphe dans toule l'étendue de la sphère et par conséquent fonction rationnelle de $\zeta$; on en conclurait que l'équation (A.) est toujours intégrable algébriquement ce qui n'est pas exact comme vous le faites voir un peu plus loin page $\mathbf{1 6 8 .}$

- A quoi tient cette contradiction? C'est à ce que les valeurs de $\zeta$ sont de 3 sortes:

I. Celles qu'on peut faire atteindre à la fontion $\frac{f(z)}{\varphi(z)}$ en faisant décrire ̀̀ la variable $z$ sur la sphère un certain contour fini un nombre fini de fois.

2. Celles qu'on peut faire atteindre à cette fonction en faisant décrire à $z$ un contour infini ou bien un contour fini un nombre infini de fois.

1 Les lettres que nous publions ici sont d'importance pour l'histoire de la thérie des fonctions fuchsiennes. Ce sont en effet ces lettres dont parle L. Fuchs dans les Nachrichten von der Königl. Gesellschaft der Wissenschaften und der G.-A.-Universität, Göttingen I882, S. 83. Gesammelte Mathematische Werke, Band II, S. 286.

2 t. 89,1880, p. I 5 I-I 69. 
3. Celles qu'on ne peut jamais faire atteindre à la fonction $\frac{f(z)}{\varphi(z)} q u e l$ que soit le contour décrit par $z$ sur la sphère.

Rien ne prouve en effet a priori qu'il n'y ait pas des valeurs de ces trois sortes et même, en général, on est certain qu'il y en a de la $2^{\text {de }}$ ou de la $3^{e}$ sorte, sans quoi, je le répète, l'équation (4.) serait intégrable algébriquement.

Mais alors il me semble qu'il faudrait encore démontrer que $z$ reste méromorphe quand $\zeta$ prend une valeur de la $2^{\theta}$ ou de la $3^{\theta}$ sorte, et que la démonstration que vous avez donnée dans votre mémoire ne s'applique qu'à celles de la I ère sorte.

On peut en effet faire plusieurs hypothèses:

I. on peut supposer que l'on n'a que des valeurs de la I ère sorte et alors $z$ est rationnel en $:-$

2. on peut supposer que l'on a des valeurs de la première et de la $2^{\circ}$ sorte et que $z$ reste monodrome quand $\zeta$ prend une des valeurs de la $2^{\text {e }}$ sorte. Dans cette hypothèse votre théorème trouverait encore son application.

3. on peut supposer que l'on a des valeurs de la I ${ }^{\text {ère }}$ et de la $2^{\text {e }}$ sorte, mais que $z$ ne revienne pas à la même valeur, quand $\zeta$ décrit un contour infiniment petit autour d'une des valeurs de la $2^{\ominus}$ sorte.

4. on peut encore imaginer que l'on ait des valeurs des trois sortes; que les

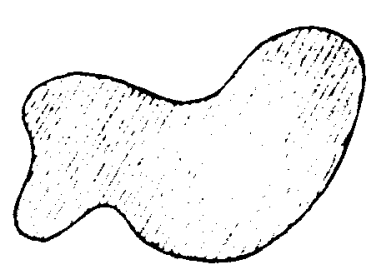
valeurs de la I ère sorte remplissent la région du plan que je couvre de hachures sur la figure, que le périmètre de cette région soit formé de valeurs de la $2^{\mathrm{e}}$ sorte; enfin que les parties extérieures à ce périmètre correspondent à des valeurs de la $3^{\mathrm{e}}$ sorte. Alors la fonction $z$ n'existe plus quand 5 sort de ce périmètre, et ne peut prendre qu'une seule valeur quand $\zeta$ reste dans ce périmètre. Alors $z$ n'est pas, à proprement parler fonction analytique de $\zeta$; mais elle est eindeutig en $\zeta$, ce qui vous suffit pour les conséquences que vous en tirez.

5. on peut imaginer que l'on ait des valeurs des trois sortes, disposées comme dans la figure ci-contre, où la région occupée par des valeurs de la $I^{\text {̀ेe }}$ sorte est couverte de hachures. Alors $z$ pourrait ne pas revenir à la même valeur quand $\zeta$ décrirait un contour tel que $H H H H$.

Enfin on pourrait encore faire mille autres hypothèses.

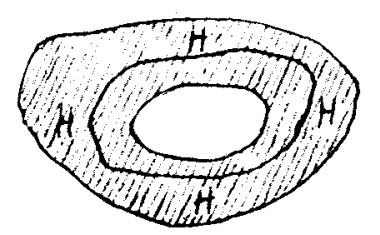

Je dois avouer, Monsieur, que ces réflexions m'ont

inspiré quelques doutes sur la généralité du résultat que vous annoncez, et j'ai pris la liberté de vous en parler, dans l'espérance que vous n'auriez pas de peine à les éclaircir. 
Mon adresse est: Henri Poincaré, Professeur à la Faculté des Sciences de Caen (Calvados).

Veuillez agréer, Monsieur le Professeur, l'assurance de ma considération respectueuse.

Poincaré.

2.

Caen, le I2 Juin I880.

Très-honoré Monsieur,

Je vous demande pardon, d'avoir tant tardé à répondre à votre aimable lettre; mais j'étais absent de Caen, lorsqu'elle est arrivée à son adresse; je l'ai reçue ce matin seulement et je l'ai lue avec le plus grand intérêt. Je vous remercie beaucoup des éclaircissements que vous avez bien voulu me donner et qui m'ouvrent des vues nouvelles sur cette question. Cependant, si je ne craignais d'abuser de votre obligeance, je prendrais la liberté d'appeler encore votre attention sur quelques points de détail, qui me semblent encore un peu obscurs.

Je suppose que sur le plan des $z$, je joigne tous les points singuliers au point $\infty$ par des coupures, puis que je fasse mouvoir $z$ dans son plan de telle sorte qu'il ne franchisse aucune des coupures. $\zeta$ va alors erfïllen dans son plan une certaine surface $F_{0}$ qui sera évidemment zusammenhangend. Faisons maintenant mouvoir $z$ dans son plan de telle sorte qu'il ne puisse franchir les diverses coupures plus de $m$ fois; $\zeta$ va rester compris dans une nouvelle surface $F_{m}$ qui sera toujours zusammenhangend. Quand $m$ augmentera la région $F_{m}$ va s'étendre de plus en plus et la surface que vous appelez $F$ n'est autre chose que la limite de $F_{m}$ pour $m=\infty$. Dire que cette surface $F^{\prime}$ ne recouvre le plan que einfach, c'est dire que, quelque grand que soit $m, F_{m}$ ne recourrira le plan que einfach.

Or cela est-il une conséquence nécessaire de votre démonstration? Il me semble qu'il faudrait pour le faire voir ajouter quelques explications. En effet, comment lorsque $m$ grandit, la région $F_{m}$ peut-elle arriver à se recouvrir ellemême? Elle peut y arriver de deux manières ainsi que l'indique la figure suivante où le trait plein représente le contour de la région $F_{m}$ et où cette région est recouverte d'une couche de hachures pendant que les parties du plan oì $\boldsymbol{F}_{m}$ se recouvre elle-même sont couvertes d'une double couche de hachures. 

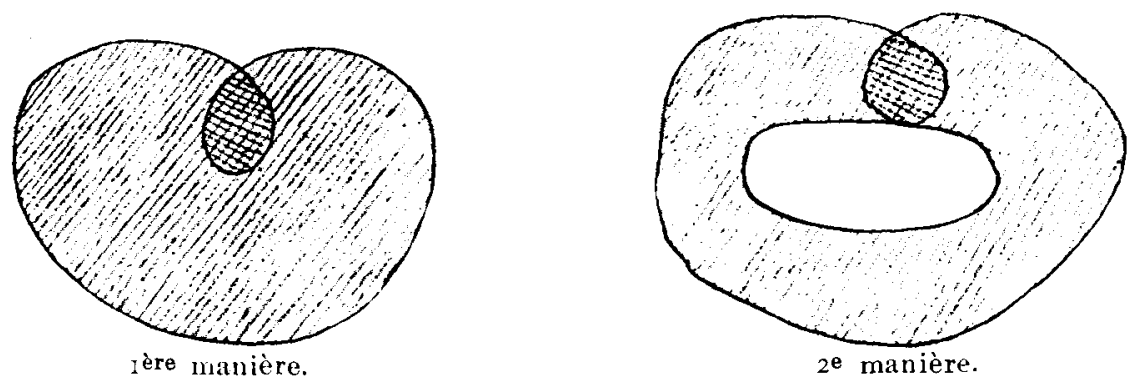

Votre démonstration, Monsieur, me paraît faire voir de la façon la plus claire, que la région $F_{m}$ ne peut se recouvrir elle-même de la $\mathrm{I}^{\text {ere }}$ manière; mais non pas qu'elle ne peut se recourrir elle-même de la $2^{\mathrm{e}}$ manière.

Je vois bien que cela est vrai lorsqu'il n'y a que deux points singuliers à distance finie. Dans ce cas j'arrive en effet, par des considérations un peu différentes à démontrer que $F_{m}$ ne peut se recouvrire elle-même ni de la $I^{\text {ère }}$ nj de la $2^{\theta}$ manière. Alors la fonction $z$ reste eindeutig dans l'intérieur de la surface $F$ qui est ici un cercle.

Mais il ne me paraît pas évident qu'il en soit de même dans le cas général, de sorte que je me demande si le théorème est encore rrai quand il $y$ a plus de deux points singuliers à distance finie.

Dans le cas où ces points singuliers ne sont qu'au nombre de deux je trouve que la fonction que vous avez définie jouit de propriétés fort remarquables et comme j'ai l'intention de publier les résultats que j'ai obtenus, je vous demanderai la permission de lui donner le nom de fonction Fuchsienne puisque c'est vous qui l'avez découverte.

Je vous demanderai aussi la permission de communiquer votre lettre à M. Hermite qui s'intéresse beaucoup à cette question.

Veuillez agréer, très honoré Monsieur, l'assurance de ma considération respectueuse.

Poincaré.

3.

Caen, le rg Juin I880.

Monsieur et cher collègue.

Je ne saurais vous dire combien je suis satisfait d'apprendre que vous avez complètement résolu le problème qui fait l'objet de notre correspondance et combien je suis désireux de recevoir l'extrajt que vous avez bien voulu me promettre et dont je vous suis bien reconnaissant d'avance.

Les conditions que vous aviez posées dans votre mémoire, pour que $z$ fût eindeutig en $\zeta$, étaient, si je me rappelle bien, les suivantes: 


$$
r_{1, i}=-\mathrm{I}+\frac{\mathrm{I}}{n_{i}}, r_{2, i}=-\mathrm{I}+\frac{2}{n_{i}} \text { ou } r_{1, i}=-\frac{\mathrm{I}}{2}, r_{2, i}=\frac{\mathrm{I}}{2}
$$

et

$$
s_{1}=\mathrm{I}+\frac{\mathrm{r}}{n}, s_{2}=\mathrm{I}+\frac{2}{n} \text { ou } s_{1}=\frac{3}{2}, s_{2}=\frac{5}{2}
$$

Or voici d'abord ce que je trouve au sujet des équations qui satisfont à ces conditions. Si on les réduit à la forme canonique, de façon à faire disparaître le terme en $\frac{d y}{d z}$, les points singuliers situés à distance finie et tels que la différence des racines de l'équation fondamentale soit I disparaissent.

Il peut arriver alors que l'on ait:

$$
s_{1}=0, s_{2}=-I \text {. }
$$

Dans ce cas on posera

$$
z-a=t^{-1}
$$

si $a$ est un des points singuliers à distance finie; puis on ramenera de nouveau l'équation à la forme canonique; le point singulier $t=0$ qui correspondrait au point singulier $z=\infty$ disparaît. Quand toutes ces opérations sont effectuées:

I. Pour tous les "points singuliers, soit à distance finie, soit à distance infinie, la différence des racines de l'équation déterminante est une partie aliquote de l'unité et est différente de $r$.

2. Le nombre des points singuliers à distance finie (qui n'ont pas disparu dans les opérations précédentes) ne peut être plus grand que 3 .

Il reste alors à considérer 4 cas.

$\mathrm{I}^{\mathrm{er}}$ cas. Le nombre des points singuliers finis est plus petit que 2. Alors $z$ est rationnel en $\zeta$.

$2^{e}$ cas. Le nombre des points singuliers est égal à 2 ; et si $\varrho_{1}, \varrho_{2}, \varrho_{3}$ sont les différences des racines des équations fondamentales déterminantes relatives à ces deux points et à $z=\infty$, on a:

$$
\varrho_{1}+\varrho_{2}+\varrho_{3}>\mathrm{I} \text {. }
$$

Alors $z$ est encore rationnel en $\zeta$.

$3^{\ominus}$ cas. Le nombre des points singuliers est 2 mais

$$
\varrho_{1}+\varrho_{2}+\varrho_{3}=\mathrm{I} \text {. }
$$

Alors $z$ est une fonction doublement périodique de $\zeta$.

$4^{\mathrm{e}}$ cas. Le nombre des points singuliers est 3 et il faut alors que la différence 
des racines de toutes les équations déterminantes soit $\frac{I}{2}$. C'est sur ce dernier cas que je prendrai la liberté d'attirer votre attention. On peut en effet former l'équation différentielle correspondante de la façon suivante; soit $A(u)$ une fonction doublement périodique de $u$ définie par l'équation différentielle

$$
\frac{d \Lambda}{d u}=\sqrt{H}=P^{3},
$$

$H$ étant un polynome du $3^{\oplus}$ degré en $\Lambda$. Posons:

$$
z=\Lambda(u), \eta=\sqrt{\frac{d \Lambda}{d u}} e^{a u}=P e^{a u}
$$

on aura

$$
\frac{d u}{d z}=\frac{I}{P^{z}}, \frac{d^{2} u}{d z^{2}}=-\frac{2 P^{\prime}}{P^{3}},
$$

et $\eta$ satisfera à l'équation différentielle

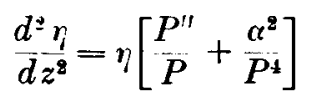

ou

$$
\frac{d^{2} \eta}{d z^{2}}=\eta\left[\frac{\frac{\mathrm{I}}{4} H H^{\prime \prime}-\frac{3}{\mathrm{I}} H^{18}+\alpha^{2} H}{H^{2}}\right] .
$$

L'autre intégrale sera

$$
\eta=P \cdot e^{-a u}
$$

d'où

$$
\zeta=e^{2 a u}
$$

Pour que $z$, c'est-à-dire $\Lambda$ fût eindeutig en $\zeta$, il faudrait que la fonction $\Lambda(u)$ admît la période $\frac{i \pi}{\alpha}$ ce qui n'a pas lieu en général.

Et pourtant si l'on pose

$$
\eta=\eta_{1} H^{-\frac{3}{4}}
$$

$\eta_{1}$ se trouve lié à $z$ par une équation différentielle linéaire (2).

L'équation (2) admet 3 points singuliers à distance finie et l'un à l'infini. Les racines de l'équation déterminante sont: 
$\mathrm{I}^{0}$. pour les points à distance finie

$$
-\frac{I}{2}=-I+\frac{I}{2} \quad \text { et } \quad 0=-I+\frac{2}{2} \text {, }
$$

$2^{o}$. pour le point à l'infini

$$
\frac{3}{2}=\mathrm{I}+\frac{\mathrm{I}}{2} \quad \text { et } \quad 2=\mathrm{I}+\frac{2}{2}
$$

L'équation satisfait donc aux conditions

$$
\begin{gathered}
r_{1}=-\mathrm{I}+\frac{\mathrm{I}}{n} \quad \text { et } \quad r_{2}=-\mathrm{I}+\frac{2}{n}, \\
s_{1}=\mathrm{I}+\frac{\mathrm{I}}{n} \quad \text { et } \quad s_{2}=\mathrm{I}+\frac{2}{n}
\end{gathered}
$$

et pourtant $z$ n'est pas eindeutig en $\zeta$, de sorte que dans ce cas particulier votre théorème me semble en defaut.

Mais ce n'est pas tout, et, si je ne craignais d'abuser de votre patience, je vous ferais part de quelques réflexions que m'a suggérées l'étude de cette question.

Les conditions que vous avez posées:

$$
\begin{gathered}
r_{1}=\frac{\mathrm{I}}{n}-\mathrm{I}, r_{2}=\frac{2}{n}-\mathrm{I} \text { ou } r_{1}=-\frac{\mathrm{I}}{2}, r_{2}=\frac{\mathrm{I}}{2}, \\
s_{1}=\mathrm{I}+\frac{\mathrm{I}}{n}, s_{2}=\mathrm{I}+\frac{2}{n} \text { ou } s_{1}=\frac{3}{2}, s_{2}=\frac{5}{2}
\end{gathered}
$$

vous les avez trouvées en cherchant à satisfaire à deux hypothèses: $I^{0} z$ devait être eindeutig en $\zeta, 2^{\circ}$ toute fonction rationnelle et symmétrique de $z_{1}$ et de $z_{2}$ devait être eindeutig en $u_{1}$ et en $u_{2}$.

Mais si l'on ne fait que la première hypothèse ( $z$ eindeutig en $\zeta$ ) ces conditions ne sont plus nécessaires. Si en effet l'objection dont je vous ai parlé dans ma dernière lettre n'existait pas, les conditions que l'on trouverait (en raisonnant tout à fait comme vous l'avez fait dans votre mémoire) seraient les suivantes: que pour tous les points singuliers la différence des racines des équations déterminantes fût une partie aliquote de l'unité. On aurait ainsi une classe d'équations beaucoup plus étendue que celle dont vous vous êtes occupé et auxquelles votre théorème s'appliquerait. Malheureusement l'objection que j'ai soulevée exige une étude plus approfondie de la question; et cette étude, je n'ai pu la faire que dans le cas où il n'y a que deux points singuliers à distance finie.: 
Soient $\varrho_{1}, \varrho_{2}, \varrho_{3}$ les différences des racines des trois équations déterminantes. Si on a:

$z$ est rationnel en $\zeta$.

$$
\varrho_{1}+\varrho_{2}+\varrho_{3}>I
$$

$\mathrm{Si}$ on $\mathrm{a}$ :

$$
\varrho_{1}+\varrho_{2}+\varrho_{3}=I
$$

$z$ est doublement périodique en $\zeta$.

Ces propriétés, je les ai déjà énoncées plus haut et d'ailleurs vous les aviez sans doute déjà découvertes.

Tant que l'on suppose

$$
\begin{gathered}
r_{1}=-\mathrm{I}+\frac{\mathrm{I}}{n}, r_{2}=-\mathrm{I}+\frac{2}{n} \text { ou } r_{1}=-\frac{\mathrm{I}}{2}, r_{2}=\frac{\mathrm{I}}{2}, \\
s_{1}=\mathrm{I}+\frac{\mathrm{I}}{n}, s_{2}=\mathrm{I}+\frac{2}{n} \text { ou } s_{1}=\frac{3}{2}, s_{2}=\frac{5}{2}
\end{gathered}
$$

on ne peut avoir

$$
\varrho_{1}+\varrho_{2}+\varrho_{3}<I \text {. }
$$

Mais si l'on se borne à la première hypothèse ( $z$ eindeutig en $\zeta$ ) $\varrho_{1}, \varrho_{2}, \varrho_{3}$ ne sont plus assujettis qu'à être des parties aliquotes de l'unité, et on peut avoir:

$$
\varrho_{1}+\varrho_{2}+\varrho_{3}<\mathrm{I}
$$

Alors $z$ n'est plus ni rationnel, ni doublement périodique en $\zeta$, mais je démontre que mon objection peut être levée et que $z$ reste eindeutig en $\zeta$. C'est cette fonction nouvelle que j'ai appelée fonction Fuchsienne et à l'aide de cette transcendante nouvelle et d'une autre qui s'y rattache j'intègre l'équation différentielle du $2^{\mathrm{d}}$ ordre à 2 points singuliers finis, non seulement quand $\varrho_{1}, \varrho_{2}, \varrho_{3}$ sont parties aliquotes de l'unité; mais quand $\varrho_{1}, \varrho_{2}, \varrho_{3}$ sont des quantités commensurables quelconques.

La fonction Fuchsienne a beaucoup d'analogies avec les fonctions elliptiques; elle n'existe que dans l'intérieur d'un certain cercle et reste méromorphe à l'intérieur de ce cercle. Elle s'exprime par le quotient de deux séries convergentes dans tout ce cercle.

Je ne sais rien au contraire sur les équations différentielles quand il y a plus de 2 points singuliers à distance finie.

Permettez-moi, Monsieur, de vous remercier de votre complaisance, de remercier aussi les équations linéaires auxquelles je dois le plaisir d'être entré en correspondance avec vous. 
Veuillez excuser la longueur de ma lettre et agréez l'assurance de ma respectueuse considération.

Poincaré.

4 .

Monsieur,

Caen, le 30 Juillet I880.

Je vous remercie beaucoup de l'envoi que vous avez bien voulu me faire de votre petit opuscule. ${ }^{1}$ Le tableau que vous donnez des intégrales de toutes les équations différentielles lève complètement tous les doutes.

C'est dans les cas III (r) et IV (I) que s'appliquait mon objection; vous envisagez en effet l'équation:

$$
\frac{d^{2} \omega}{d z^{2}}+\frac{\mathrm{r}}{2} \frac{d \log R}{d z} \frac{d \omega}{d z}+\frac{\pi^{2}}{\Omega^{2}} \frac{\mathrm{I}}{R} \omega=0
$$

pour laquelle votre théorème est évidemment vrai; mais si au lieu de $\frac{\pi^{2}}{\Omega^{2}}$ vous aviez pris un coëfficient numérique quelconque $\alpha$, le théorème se serait trouvé en défaut, quoique les conditions que vous aviez énoncées primitivement et qui sont relatives aux racines des équations déterminantes eussent continué à être remplies. Comme rous aviez négligé d'énoncer cette condition supplémentaire, relative à la valeur du coëfficient numérique de $\frac{I}{R}$, je m'y étais laissé tromper et vous voudrez bien m'en excuser.

Permettez-moi d'insister sur les fonctions auxquelles j'ai donné votre nom et que j'ai rencontrées dans ces recherches.

Ces fonctions présentent avec les fonctions elliptiques les plus grandes analogies et sont susceptibles d'être représentées par le quotient de deux séries convergentes, et cela d'une infinité de manières. Parmi ces séries, il y en a qui sont des séries entières et qui jouent le rôle de fonction Théta.

Elles sont convergentes dans toute l'étendue d'un certain cercle et, en debors de ce cercle elle cessent d'exister, ainsi que la fonction Fuchsienne elle-même.

En dehors de ces fonctions, il en est d'autres qui jouent le même rôle que les fonctions Zéta dans la théorie des fonctions elliptiques et grâce auxquelles j'intègre toutes les équations différentielles linéaires d'ordre quelconque à coëfficients rationnels toutes les fois qu'il n'y a que deux points singuliers à distance

1 Voir la note page 186. 
finie et que les racines des trois équations déterminantes sont commensurables. J'ai imaginé aussi des fonctions qui sont aux fonctións Fuchsiennes ce que les fonctions abéliennes sont aux fonctions elliptiques et grâce auxquelles j'espère intégrer toutes les équations linéaires quand les racines des équations déterminantes seront commensurables.

Enfin des fonctions tout à fait analogues aux fonctions Fuchsiennes me donneront, je crois, les intégrales d'un grand nombre d'équations à coëfficients irrationnels.

Veuillez, Monsieur, agréer encore une fois mes remerciements, ainsi que l'assurance de ma considération la plus distinguée.

Poincaré.

5 .

Caen, le 20 Mars I88I.

Monsieur,

Je vous remercie beaucoup du mémoire que vous avez eu la bonté de m'envoyer et que j'ai lu avec le plus grand intérêt.

J'ai continué à m'occuper des fonctions auxquelles j'ai donné votre nom et j'espère publier prochainement mes résultats. Ces fonctions comprennent comme cas particulier les fonctions elliptiques d'une part, et d'autre part la fonction modulaire. Ces fonctions et d'autres que j'ai appelées zétafuchsiennes permettent d'intégrer:

$I^{\circ}$. Toutes les équations différentielles linéaires à coëfficients rationnels qui ne présentent que trois points singuliers, deux à distance finie et l'un à l'infini.

$2^{0}$. Toutes les équations $\mathrm{du} 2^{\mathrm{d}}$ ordre à coëfficients rationnels.

$3^{\circ}$. Un grand nombre d'équations de divers ordres à coëfficients algébriques.

Venillez agréer, Monsieur, l'assurance de ma considération la plus distinguée. 